\title{
Coping Strategies and Brain Activity in Table Tennis Players Faced with Discrepancies between Predicted and Actual Outcomes
}

\author{
Yinyue Wang, Qiwei Zhao, Xia Shan, Yingzhi Lu \\ School of Psychology, Shanghai University of Sport, Shanghai, China
}

Correspondence: Yingzhi Lu, School of Psychology, Shanghai University of Sport, No. 399 Changhai Road, Yangpu District, Shanghai 200438, China. Email: luyingzhi@sus.edu.cn

\begin{abstract}
Prior studies have shown that athletes possess an excellent ability for action anticipation. However, it is not clear how athletes manage to regulate their behaviors when predicted outcomes deviate from actual outcomes. Based on Bayes theory and the predicted response outcome (PRO) model, the current study explored this question using novice and expert table tennis players. Discrepancies between predicted outcomes and actual outcomes were varied into seven conditions. We first examined the differences between experts and novices in a congruence discrimination task and confirmed that experts were better than novices at identifying discrepancies. Subsequently, EEG technology was used to further explore the specific behavior and neural activity in experts during discrepancy processing. Reaction time and theta oscillations gradually increased as the size of discrepancy between the predicted and the actual outcomes increased. These results suggest that experience promotes the monitoring of discrepancies between predicted and actual outcomes in athletes leading to flexibility in coping with varying degrees discrepancy.
\end{abstract}

Keywords: discrepancy, predicted outcomes, expertise, theta oscillation 


\section{Introduction}

Anticipation of an opponent's actions is a critical skill for many athletes, especially in sports involving high time pressure (Rowe, Poulter, \& McKenna, 2009; Wims-hurst, Sowden, \& Wright, 2016). Previous studies have revealed that compared with nonplayers or novice players, high-performance players can better predict the outcomes of another player's sequential movements (e.g., Denis, Rowe, Williams, \& Milne, 2017; Ward, Williams, \& Bennett, 2002). The high accuracy in action prediction helps highperformance players to prepare for their next movement. Furthermore, some studies have also indicated that even in unpredictable situations, expert players maintain superior performance compared to non-players or novice players (Michael, Daniel, Robin, \& Bruce, 2013). Prior studies have confirmed that high-performance players utilize kinematic information and previously learned information to effectively anticipate actions (Murray \& Hunfalvay, 2016; Vaeyens, Lenoir, Williams, \& Philippaerts, 2007). In addition, high-performance players must also effectively compare predicted outcomes with actual outcomes, and understand possible discrepancy between the two. However, how expert players process these discrepancies remains unclear.

Frequently, within all sports, a predicted outcome does not match the actual outcome. For example, in table tennis, the ball may make contact with the net, followed by an unexpected trajectory change. Studies on expert players have proposed that longterm dedicated practice enables athletes to develop specific cognitive processes for responding to unpredicted events. A recent study tested football players' sensitivity to deceptive actions in a semi-immersive lab setting, asking participants to judge which direction life-size opposing players would move. High-skilled and recreational rugby players were equally vulnerable to deception. However, high-skilled players were faster to discover that the actual situation did not match their expectation, allowing them to respond more effectively to deceptive behavior (Warren-Westgate, Jackson, Blenkinsop, \& Hiley, 2021).

Compared with non-players, experts show higher discrimination accuracy, suggesting that experts have the ability to detect discrepancy between predicted outcomes and actual outcomes (e.g., Rowe et al., 2009; Ward et al., 2002). In addition, these 
studies revealed that experts reacted faster than non-players, especially in deceptive situations. Combined with higher discrimination ability, these results suggest that experts have effective coping strategies for processing the discrepancy between predicted and actual outcomes.

Consistent with this line of research, a previous study (Hung, Spalding, Santa Maria, \& Hatfield, 2004) asked table tennis players and non-players to judge a target position in situations where a cue position matched or was mismatched with the target. Compared with non-players, expert players displayed faster reaction times and higher accuracy in the mismatched conditions. Furthermore, expert players had a larger range of visual attention, suggesting that compared to novices, experts attend visually more to the unexpected stimuli while simultaneously preparing a motor response in the direction of the cued or expected stimuli (Hung et al., 2004). This diversified attentional strategy likely contributes to an expert's sustained heightened reactive capacity, while responding quickly to both the matched and mismatched stimuli. Generally, these studies suggest that experts develop specific cognitive processing skills to detect and cope with discrepancy between predicted and actual outcomes.

However, these studies do not take into consideration sport scenes, which the discrepancy between expected and actual outcomes were more than only none (i.e., match) and all (i.e., mismatch). For example, in table tennis, the possible landing position of the ball is not evenly distributed on the table, and the landing point may be near a boundary line which is the most difficult place for players to return. Therefore, the discrepancies between prediction and actual outcomes cannot be simply divided into matched or mis-matched. The prediction of the landing point of the ball is not one specific position, but rather a range. According to Bayes theory, the discrepancy between a predicted outcome and the actual outcome is a gradual increase in change (Körding, 2007). As such, it is important to manipulate a gradual changing discrepancy to improve the ecological validity in an action anticipation study. Consequently, in the current study, we designed seven conditions, each changing gradually, from outcomes that were the same as those predicted, to outcomes that were opposite of those predicted (see method for details). 
In addition to behavioral analysis, it is important to understand the underlying brain mechanisms responsible for successful processing and responding to the gradually changing discrepancy during action anticipation. In previous studies ( $\mathrm{Lu}$ et.al., 2019; Zhao et al., 2021), we found that EEG theta activity over the mid-frontal cortex was involved in the evaluation of discrepancy monitoring in experts and novices. Traditional reinforcement learning postulates that the brain acquires skills by learning associations between stimuli and corresponding correct outcomes (Scheffers \& Coles, 2000). In particular, if an athlete's anticipation of an action is correct, the correct outcome reinforces the athlete's anticipation of the action in the future. In contrast to reinforcement learning, the predicted response outcome (PRO) suggests that the individual learns the association between prediction and possible outcomes, including discrepancies (Alexander \& Brown, 2012). Furthermore, the PRO model posits that neurons in the medial prefrontal cortex code learned probabilities of potential action outcomes and these neural signals attenuate upon the occurrence of an expected outcome but are amplified upon the occurrence of an unexpected outcome (Alexander \& Brown, 2011).Our previous study found a larger mid-frontal theta oscillation when an outcome is not consistence with kinematic information (i.e., incongruent) than when an outcome matches kinematic information (Lu et.al., 2019).

Prior studies have found that incongruent information induces larger theta oscillations of the medial prefrontal cortex (mPFC), compared to those produced during congruent conditions (Cavanagh and Frank, 2014; Cohen, 2014). Therefore, it is speculated that theta oscillations are involved in the cognitive processing of action to monitor discrepancies between expected and actual outcomes (Cavanagh, Cohen, \& Allen,2009). According to the PRO model and Bayes theory, we hypothesized that theta oscillations would gradually increase as athletes detect larger discrepancies between predicted and actual outcomes.

The present study explores how table tennis experts process the discrepancy between predicted and actual outcomes. Experiment 1 explores the ability of novices and experts in detecting discrepancies between kinematic information from the server and the ball flight information using manipulated videos. Based on results from Experiment 
1, Experiment 2 investigates the underlying neural mechanism used by experts in detecting discrepancy. Experts were asked to predict the ball landing location according to the server's kinematic information, by indicating the actual location as quickly as possible when the outcome was presented. Based on the above, we hypothesized that compared with novices, experts would use superior cognitive and coping strategies when presented with discrepancies. Second, we hypothesized that at the neural level, theta oscillation activity in the mPFC would increase as discrepancy increased.

\section{Experiment 1}

\subsection{Methods}

\subsubsection{Participants}

A total of 40 volunteers participated in Experiment 1, including 20 experts (10 males and 10 females) and 20 novices ( 9 males and 11 females). Experts were required to have more than eight years of professional training experience in table tennis. Novices had not participated in any professional training related to table tennis and were not in the habit of watching table tennis matches. There was no difference in sex $\left(t_{(38)}=1.907, p=.064,95 \%\right.$ confidence interval $\left.[-0.077,2.577]\right)$ or in age $\left(\chi^{2}=0.1, p=.752\right)$ between the two groups (Table 1). All participants were right-handed, in good health, without mental disease, with normal or corrected vision, and without color blindness or color weakness. Participants had never previously participated in similar experiments or volunteered to participate in the current experiment. After completion of the experiment, participants were given a monetary reward. The Ethics Committee of Shanghai University of Sport approved all experimental procedures (Approval No.102772020RT111). 
Table 1. Demographic information and table tennis experience of participants in Experiment 1

\begin{tabular}{lll}
\hline Group & Novice & Expert \\
\hline Sample size & 20 & 20 \\
Sex (female:male) & $11: 9$ & $10: 10$ \\
Age (year) & $20.60 \pm 2.23$ & $19.35 \pm 1.89$ \\
Years of training (year) & $\mathrm{n} / \mathrm{a}$ & $10.40 \pm 3.96$ \\
Frequency ${ }^{\mathrm{a}}$ (times/week) & $\mathrm{n} / \mathrm{a}$ & $3.43 \pm 2.33$ \\
Duration $^{\mathrm{a}}$ (hr) & $\mathrm{n} / \mathrm{a}$ & $2.85 \pm 1.24$ \\
\hline
\end{tabular}

${ }^{\text {a } F r e q u e n c y ~ r e f e r s ~ t o ~ t h e ~ n u m b e r ~ o f ~ t a b l e ~ t e n n i s ~ s e s s i o n s ~ p e r ~ w e e k, ~ d u r a t i o n ~ r e f e r s ~ t o ~ t h e ~}$ duration of each session. Means and standard deviations are displayed.

\subsubsection{Stimuli}

A total of 21 videos were recorded using Canon 5D mark III (resolution, 1280× 720 pixels, 30 frames per second) positioned horizontally $40 \mathrm{~cm}$ from the midline on the receiver's side. Players from the China Table Tennis Academy were asked to serve balls to 7 different landing points, zone 1 , zone 2 , zone 3 , zone 4 , zone 5 , zone 6 , and zone 7 (the horizontal width of the table was divided into these 7 zones. See Figure 1a). The video was processed by Adobe Premiere software, and then exported as continuous images at 25 frames per second (resolution, $640 \times 360$ pixels). A complete serving video was comprised of 36 consecutive images (Figure 1b). The server's kinematic information was registered as the 18 frames prior to the "racquet-ball" contact moment, while the 17 frames after contact were recorded as the ball flight information. The server's face was blurred in all videos to eliminate facial information interference. 
Each kinematic stimulus (i.e., the racquet-ball contact moment plus the preceding 18 images) was followed by seven different ball flight outcomes (i.e., 17 images following the racquet-ball contact moment). As such, there were seven degrees of discrepancy between the predicted ball flight trajectory and observed ball flight trajectory. Taking the serving video with landing point in zone 1 as an example, an actual landing point in zone 1 had a discrepancy of $0 \%$; while an actual landing point in zone 2 had a discrepancy of $16 \%$, and so on. In this manner, seven degrees of discrepancy existed $(0 \%, 16 \%$, $33 \%, 50 \%, 66 \%, 83 \%$, and 100\%) (Figure $1 \mathrm{~b}$ ).

(a)

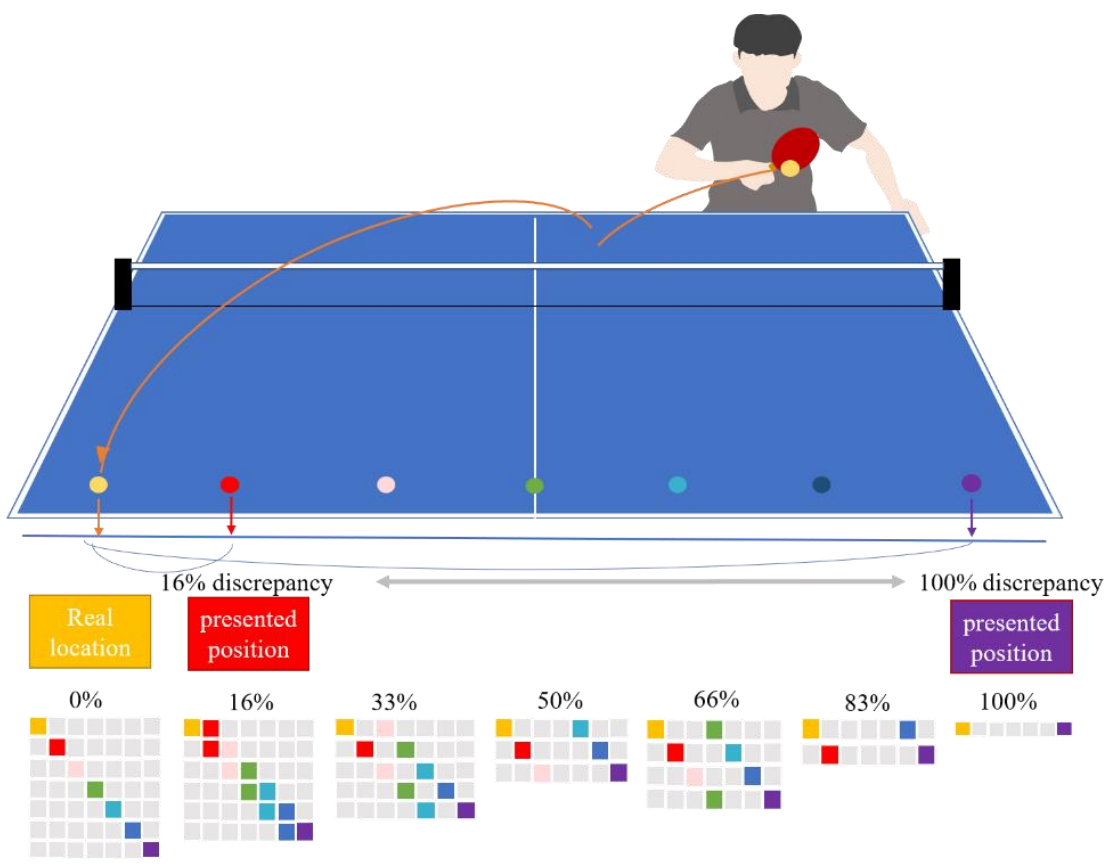

(b)

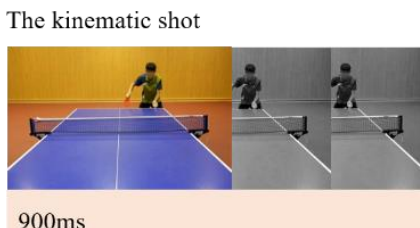

(18 pictures)
Racquet-ball contact The ball flight shot

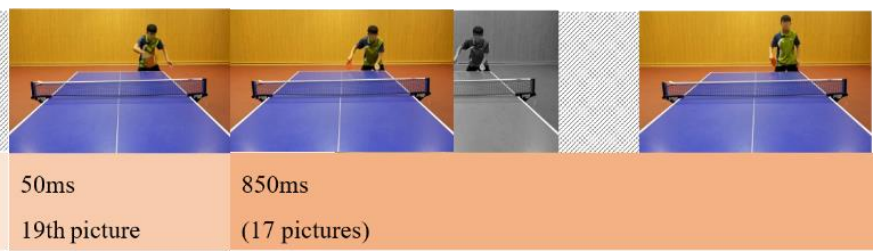

Figure 1(a) Calculation of discrepancies. Seven colored balls represent seven zones. If the actual landing point was in zone 1 (the yellow ball) and the presented landing point was in zone 1, the discrepancy was " $0 \% "$, i.e., there was no deviation. If the actual landing point was in zone 1 and the 
presented landing point was in zone 2 (the red ball), the discrepancy was "16\%". Discrepancies continue to rise as the presented landing point moves farther from the actual landing point. All combinations of the seven deviations are shown at the bottom of the diagram. (b) Stimuli. Each video was $1,800 \mathrm{~ms}$ and started with the player preparing to serve and ended with the player completing the serve. The trajectory of the ball after racquet-ball contact was deleted and replaced with the trajectory of the ball from another video.

\subsubsection{Task and procedure}

To become familiar with the experimental process, each participant completed 14 practice trials including 2 trials at each discrepancy condition (0-100). The formal experiment consisted of 4 blocks of 50 trials each, resulting in a total of 200 trials. Each trial was presented randomly. The participants were asked to judge the consistency of the kinematic information and ball flight information. If the predicted landing location was anticipated according to the server kinematics, it was recorded as a "consistent" condition (i.e., $0 \%$ discrepancy); otherwise, it was recorded as an "inconsistent" condition (i.e., $16 \%, 33 \%, 50 \%, 66 \%, 83 \%$ and $100 \%$ discrepancy). The participants were asked to use their personal experiences to judge the kinematic information of the server, and to press the "inconsistent" or "consistent" key as accurately as possible without time limitation.

Each trial started with a fixation crosshair in the center of the screen that lasted $1,000 \mathrm{~ms}$, followed by trial videos $(1,800 \mathrm{~ms}$ each $)$. At the end of each trial video, the prompt, "please press the key" was displayed. Participants were instructed to press the "J" key if they thought the video was consistent and the "F" key if they thought the 
video was inconsistent. Prior to the experiment, subjects were asked to place their left index finger on the "F" key and their right index finger on the "J" key. Each block lasted approximately three minutes, with a 1-minute rest period between blocks. After the experiment, participants were asked to complete a visual analog scale (VAS) about the difficulty of the task and how much they relied on editing information (See the attachment).

\subsubsection{Data and statistical analyses}

For each subject, the number of correct responses under each condition was collected to calculate the proportion of classified disguised throws (PCDT), which represents the proportion of trials identified as discrepancy in all trials (Helm, Caal-Bruland, Mann, Troje, \& Munzert, 2019).

In two-alternative forced-choice (2AFC) tasks, accuracy percentage can conflate bias with decision, and as such, we used a standard adaptation of the 2AFC task (Macmillan \& Kaplan, 1985; Vicario, Makris, \& Urgesi, 2016) by calculating and analyzing d-prime (d'). For the consistent trials, the correct response was regarded as a "hit", while an incorrect response was regarded as a "miss"; for the inconsistent trials, the correct response was regarded as a "correct rejection" and an incorrect response was regarded as a "false-alarm". The d' values were calculated by transforming the response proportions to $\mathrm{z}$ scores, and then subtracting the hit rate $\mathrm{z}$ score from the false-alarm $\mathrm{z}$ score. Hence, the higher the d' score, the better participants were able to detect a discrepancy in the kinematic patterns of the videos.

All data analyses were performed in SPSS 20.0 (IBM, Somers, USA). Mixed 
analysis of variance (MANOVA) was used to test PCDT with Expertise as a betweensubjects factor and Degree of discrepancy as a within-subject factor. Post hoc tests were corrected using Bonferroni corrections. The difference in d' between the groups was determined by an independent sample t-test. Partial eta-squared $\left(\eta_{\mathrm{p}}^{2}\right)$ was used to represent the effect size for the MANOVA, and a d-value was used as the effect size for the t-test. Bayes test was used as an auxiliary means to test the effect. Data from three expert participants were excluded due to missing VAS questionnaires and therefore, the independent sample t-test was conducted on 17 experts and 20 novices (See the attachment).

\subsection{Results}

The MANOVA analysis on PCDT showed main effects of Expertise $\left(F_{(6,228)}=11.985, P=.001, \eta_{\mathrm{p}}^{2}=.24, \mathrm{BF}_{10}>100\right)$ and Degree of discrepancy $\left(F_{(6,228)}=67.775\right.$, $\left.P<.001, \eta_{\mathrm{p}}^{2}=.641, \mathrm{BF}_{10}>100\right)$. The interaction between Expertise and Degree of discrepancy was also significant $\left(F_{(6,228)}=7.221, P<.001, \eta_{\mathrm{p}}^{2}=.160, \mathrm{BF}_{10}>100\right)$. The pairwise comparisons of the interaction showed that experts and novices differed significantly at the $0 \%, 66 \%$, and $100 \%$ discrepancy conditions $\left(P_{0 \%}=.011, P_{66 \%}=.050\right.$, $\left.P_{100 \%}<.001\right)$. The difference between experts and novices was not significant at other discrepancy conditions (Table 2). 
Table 2. Pairwise comparison of PCDT

\begin{tabular}{lrrrr}
\hline Degree of discrepancy & $\begin{array}{r}\text { Experts }(\mathrm{n}=20) \\
\text { Mean } \pm \mathrm{SD}\end{array}$ & $\begin{array}{r}\text { Novices(n=20) } \\
\text { Mean } \pm \text { SD }\end{array}$ & p-value & $\begin{array}{c}95 \% \text { Confidence } \\
\text { Interval }\end{array}$ \\
\hline $0.210 \pm 0.121$ & $0.318 \pm 0.133$ & 0.011 & {$[-0.189,-0.026]$} \\
$16 \%$ & $0.245 \pm 0.131$ & $0.292 \pm 0.114$ & 0.233 & {$[-0.126,0.032]$} \\
$33 \%$ & $0.356 \pm 0.122$ & $0.359 \pm 0.110$ & 0.929 & {$[-0.078,0.071]$} \\
$50 \%$ & $0.349 \pm 0.116$ & $0.305 \pm 0.112$ & 0.230 & {$[-0.029,0.117]$} \\
$66 \%$ & $0.501 \pm 0.133$ & $0.409 \pm 0.102$ & 0.050 & {$[-0.003,0.187]$} \\
$83 \%$ & $0.566 \pm 0.148$ & $0.505 \pm 0.164$ & 0.164 & {$[-0.026,0.149]$} \\
$100 \%$ & $0.702 \pm 0.188$ & $0.433 \pm 0.164$ & $<0.001$ & {$[-0.399,-0.174]$} \\
\hline
\end{tabular}

For the experts, there were significant differences between accuracy at the $16 \%$ and $33 \%$ discrepancy conditions $(P=.016,95 \% \mathrm{CI}[-0.197,-0.024])$, between the $50 \%$ and $66 \%$ conditions $(P=.001,95 \% \mathrm{CI}[-0.261,0.044])$, and between the $83 \%$ and $100 \%$ discrepancy conditions $(P=.025,95 \% \mathrm{CI}[-0.275,-0.033])$. For the novices, there was a significant difference only between the $50 \%$ and $66 \%$ discrepancy conditions $(t=-3.781$, $P=.001,95 \%$ CI $[-0.162,-0.046]$ ) (Figure 2a). The Bayes test results showed strong evidence for a difference between the $50 \%$ and $66 \%$ discrepancy conditions. d' was significantly greater for experts than novices, indicating that experts have a higher discrimination ability of discrepancy than novices $(P<.001$, Table 3 , Figure $2 b)$.

Table 3. Independent sample t-test results of d'

\begin{tabular}{|c|c|c|c|c|c|c|c|}
\hline & $\operatorname{Experts}(n=17)$ & Novices(n=20) & $\mathbf{t}$ & Cohen's d & 95\% Confidence & p-value & $\mathbf{B F}_{10}$ \\
\hline & Mean \pm SD & Mean \pm SD & & & Interval & & \\
\hline d' & $0.738 \pm 0.418$ & $0.167 \pm 0.389$ & 4.471 & 1.414 & {$[0.313,0.830]$} & $<.001$ & $>100$ \\
\hline
\end{tabular}




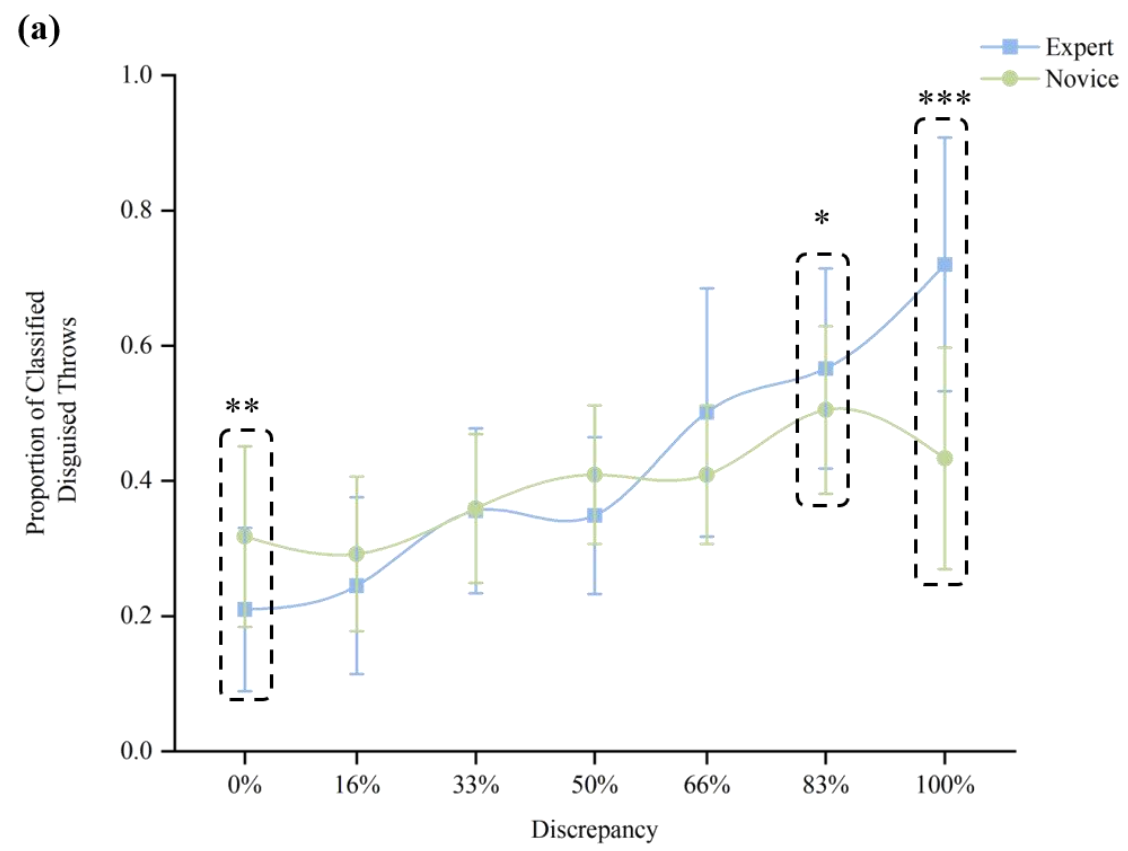

(b)

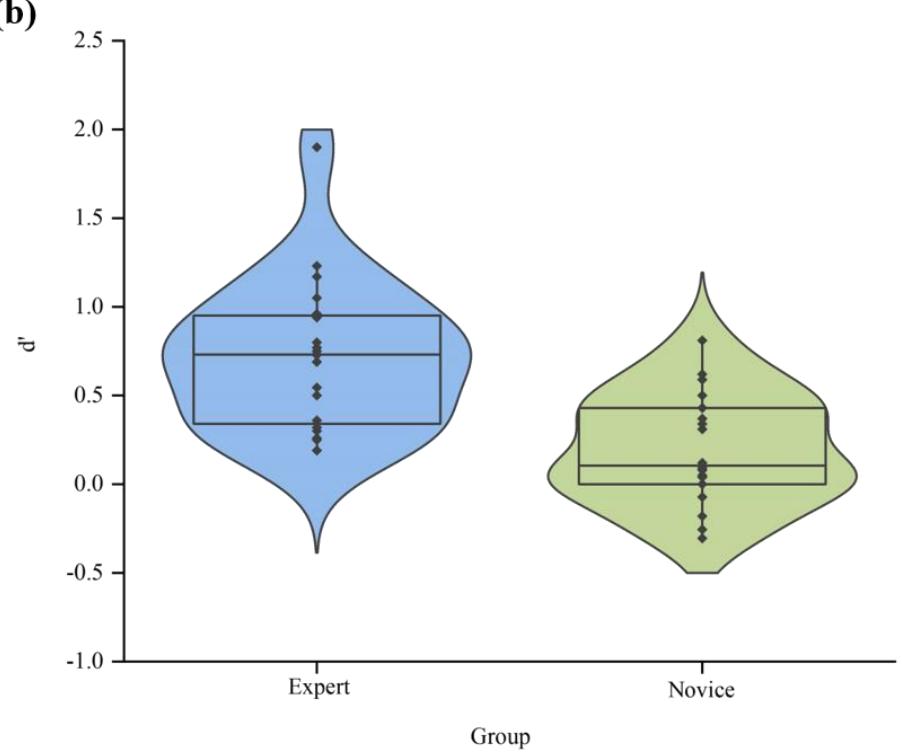

Figure 2 (a) PDCT of experts and novices under different discrepancy conditions. Blue squares represent experts and green dots represent novices. Error bars represent standard error of the mean. (b) Expert and novice d'. Error bars represent standard error of the mean. ${ }^{*} P<0.05, * * P<0.01$, $* * * P<0.001$ 


\subsection{Discussion of Experiment 1}

Compared to novices, the experts showed a higher PDCT under discrepancy conditions, especially when the discrepancy was larger than 50\%, while the PDCT was lower in experts compared to novices under the $0 \%$ discrepancy condition. These results indicate that compared to novices, experts are better at action anticipation. However, this skill was only apparent when the discrepancy was large. Prior studies have indicated that highly skilled individuals can predict outcomes more accurately than novices account of the internal model (Calvo-Merino, et.al.,2005). Novices showed a similar PDCT at all discrepancy conditions, suggesting that their sensitivity to discrepancy was restricted by their anticipation ability.

Interestingly, the PDCT of novices only differed between the $50 \%$ and $66 \%$ discrepancy conditions, suggesting that novices classify all discrepancies into two categories, consistent or inconsistent. However, experts showed different PDCT values between $16 \%$ and $33 \%, 50 \%$ and $66 \%$, and $83 \%$ and $100 \%$, indicating that experts are more sensitive to subtle discrepancies than the novices. These results are consistent with the idea that experts are flexible in their processing of predicted outcomes so that they may better adapt to a rapidly changing environment (Alexander \& Brown, 2011; Körding, 2007).

Experiment 2 was conducted to further investigate the sensitivity of perceptual discrepancy in experts. EEG technology was used to explore the dynamic brain activity, especially theta oscillations during discrepancy discrimination tasks. Our prior study found that mid-frontal theta oscillations were sensitive to the consistency between 
predicted and observed outcomes in experts (Cohen et al., 2014; Lu et al., 2019). Prior studies have shown that athletes who play ball sports, such as table tennis, have special cognitive processes to cope with unexpected events, thus reducing cognitive cost to ensure that reaction time is not delayed (Benguigui \& Ripoll, 1998; Runigo, Benguigui, \& Bardy, 2005). Therefore, in Experiment 2 we hypothesized that at the behavioral level, reaction time to judge the observed landing zone would be different with increasing discrepancies, since the perceptual data in Experiment 1 showed differences in four ranges (i.e., no discrepancy, between $16 \%$ and $33 \%, 50 \%$ and $66 \%$, and $83 \%$ and $100 \%$.).

\section{Experiment 2}

\subsection{Methods}

\subsubsection{Participants}

Thirty-two table tennis players (17 males and 15 females) participated in Experiment 2 . The participants were recruited from the college table tennis team with the following inclusion criteria: (1) must have at least 6 years of professional training experience; (2) must practice more than two days a week, and more than two hours each practice session during the last 3 years. Of the selected subjects, $56.25 \%$ qualified as National Players at the Second Grade or above. Participants were all right-hand dominant and had normal or corrected-to-normal vision. None of the participants reported a history of neurological, psychiatric, or other major medical problems. Participants were paid for their participation and signed a consent form before taking part in the study. 
The experimental procedures were approved by the Ethics Committee of the Shanghai University of Sport.

Table 4. Demographic information and table tennis experience of participants in Experiment 2

\begin{tabular}{lccccc} 
Sample size & \multicolumn{1}{c}{ Sex } & Age & Years of training & Frequency $^{\mathbf{a}}$ & Duration $^{\mathbf{a}}$ \\
& $($ female:male) & $($ years $)$ & $($ year $)$ & (times/week) & (hr) \\
\hline 32 & $17: 15$ & $21.36 \pm 2.74$ & $9.41 \pm 3.83$ & $2.69 \pm 1.23$ & $2.28 \pm 0.72$ \\
\hline
\end{tabular}

${ }^{a}$ Frequency refers to the number of table tennis sessions per week, duration refers to the duration of each session. Data are listed as mean \pm standard deviation.

\subsubsection{Stimuli}

The video used in Experiment 2 was the same as that used in Experiment 1, but occlude 6 frames after the "racquet-ball contact" in each video. As such, each video takes the "racquet-ball contact" frame as the benchmark, and selected 18 pictures forward (showing the server's kinematic information for valid action anticipation), then occlude the next six frames and the following 11 frames (showing the ball flight trajectory). As in experiment 1 , the trajectory of the ball was manipulated into seven discrepancy conditions (i.e., $0 \%, 16 \%, 33 \%, 50 \%, 66 \%, 83 \%$, and $100 \%$ ).

\subsubsection{Task and procedure}

Prior to the formal experiment, a discriminative reaction task was conducted to eliminate the influence of response key position. Reaction times to the 7 landing positions were recorded for use as a baseline for analyses. The discriminative reaction task started with a fixation point for $1,000 \mathrm{~ms}$, followed by a table tennis picture with a ball in one of the 7 zones. The participants were asked to press the corresponding number key immediately to identify the position of the ball (Figure 3b). The participants were 
asked to put their index finger on the " 5 " key as preparation and asked to complete their response within 1,100 ms. The discriminative reaction task included 3 blocks and each block contained 70 trials, with 10 trials for each of the 7 discrepancy conditions which were presented randomly.

The formal experiment consisted of 5 blocks each consisting of 70 experimental trials and 15 detection trials. The participants were asked to make a prediction within $1,000 \mathrm{~ms}$ about the ball's landing position after the kinematic shot. Participants were asked to immediately press the corresponding key to identify the ball landing position. In the detection trials, instead of the ball flight shot, the participants were asked to press the predicted landing position after the occlusion. The response keys were the same as the above-described discrimination reaction task and the experimental procedure is shown in Figure 3a. A VAS questionnaire (see Supplementary file) was completed after the experiment. The entire experiment lasted approximately 40 minutes. 
(a)

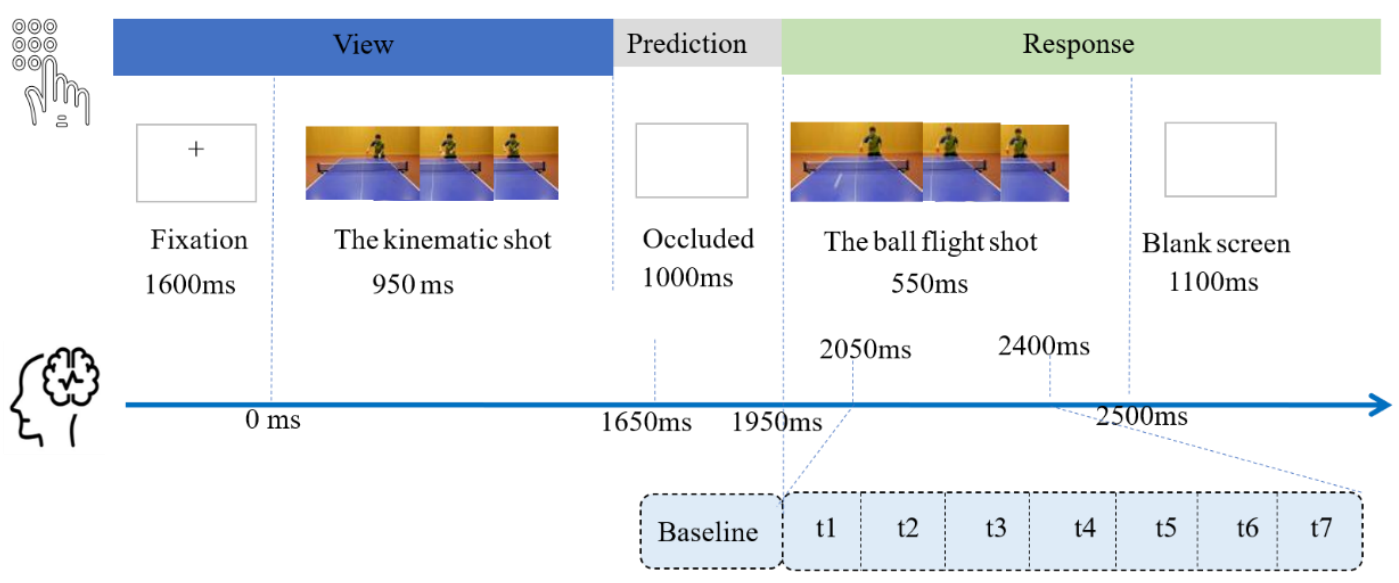

(b)

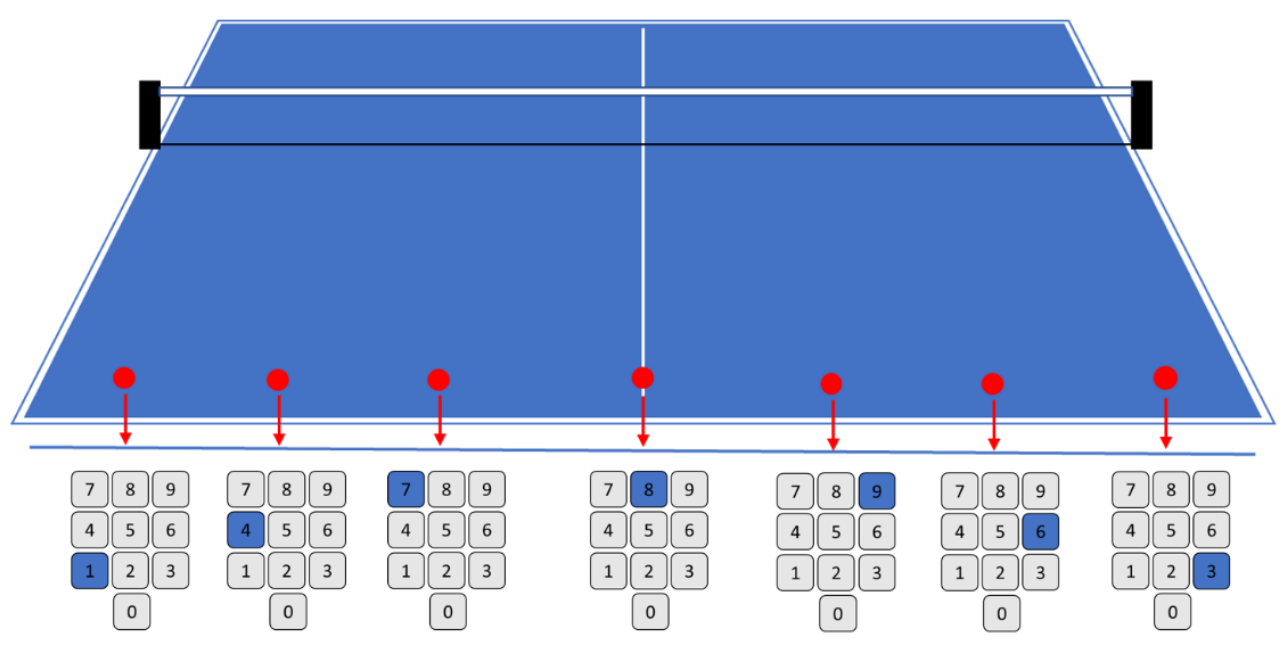

Figure 3 (a) A single trial procedure for Experiment 2. Each video was 2,450 ms. The video started with the serve, followed by $900 \mathrm{~ms}$ to racquet-ball contact, followed by a 1,000 ms blank screen, and finally by the trajectory of the ball. The trajectory of the ball after racquet-ball contact was deleted and replaced with the trajectory of the ball from another video. The segmentation of EEG data is shown at the bottom of the figure. The analysis time frame was from 2,050 to 2,400 milliseconds, with a total of 350 milliseconds, divided into seven periods. (b) The position of the ball and the corresponding buttons for the discriminative reaction task and Experiment 2. 


\subsubsection{EEG data recording}

Electroencephalograph (EEG) activity was collected from 64-channel scalp electrodes according to the International $10-20$ system at a sampling rate of $1,000 \mathrm{~Hz}$ and with the $\mathrm{AFz}$ point as the grounding electrode and the $\mathrm{FCz}$ point as the online reference electrode using BrainVision Recorder (version 2.0, Brain Products GmbH, Germany). A horizontal electrooculogram (HEOG) was placed on the right outer canthus and the vertical electrooculogram (VEOG) was placed $1 \mathrm{~cm}$ below the left orbit. Electrode impedance was maintained below $10 \mathrm{k} \Omega$.

\subsubsection{Data and statistical analyses}

Behavioral analysis

The discriminative reaction task revealed that different number key positions affected response time. The response to pressing the "1" key was significantly shorter than most other keys (Attachment), while the response times to pressing the "7" and "9" keys were the longest. To eliminate this effect, the reaction time in Experiment 2 was calculated by subtracting the reaction time in the discrimination task from the reaction time in the formal task. Based on the results from Experiment 1, in Experiment 2 we combined similar discrepancies, forming four degrees of discrepancy: little-to-no discrepancy (i.e., $0 \%$ and 16\%), small discrepancy (i.e., 33\% and 50\%), large discrepancy (i.e., 66\% and 83\%), and inconsistent (i.e., 100\%). The accuracy and reaction times in these four discrepancies were compared using a repeated measures ANOVA.

EEG data analysis

EEG data were preprocessed by MATLAB (R2016a, the MathWorks, Inc.; Natick, 
MA). First, the EEG data sets were visually inspected for each channel and participant. Second, a Butterworth filter for frequency was used with a high cutoff of $1 \mathrm{~Hz}$ and a low cutoff of $30 \mathrm{~Hz}$. Next, the signals were re-referenced to the whole brain average, and the online reference was recovered to FCz. The appearance of the stimulus video was set as time 0 , while the data after re-reference was segmented starting $1,650 \mathrm{~ms}$ to 2,500 ms after video onset. The next step was ocular correction by referring two EOG channels and calculating [yi=xi-mean(bl)] (Romain \&Arnaud, 2011).

A continuous wavelet transform (CWT) was used for the time-frequency decomposition of the segmented EEG data (i.e. 1,650 ms to 2,500 ms) for the frequency range between 1 and $30 \mathrm{~Hz}$ in MATLAB. A complex Morlet wavelet was selected as the mother wavelet, with a time decay parameter of $1.0 \mathrm{~s}$ and a center frequency of $1.5 \mathrm{~Hz}$. The baseline was corrected using the 1,650 to 1,950 ms segment using ERSP\% (a baseline correction method form Event-related spectral perturbation): [yi=(ximean(bl)/mean(bl))] (Romain \&Arnaud, 2011). The power spectrum of the CWT at each frequency was separately normalized with respect to its corresponding mean power during the baseline period (i.e., 1650 to $1950 \mathrm{~ms}$ ), by which the transient changes in the power at certain frequencies could be observed.

We selected points of interest (POIs) to detect theta oscillation activity with the following specifics:

(1) Frequency of 4 to $8 \mathrm{~Hz}$ (Guy, et al., 2016)

(2) Eleven consecutive 50-ms epochs, from 1,950 to 2,500 ms (Lu et.al.,2019). The time-frequency diagram showed intense brain activity between 2,050 and 
2,400 ms, and as such, this time window, split into seven segments, was selected for data analysis.

(3) Three EEG electrodes along the fronto-centro-parietal midline ( $\mathrm{FCz}, \mathrm{Cz}, \mathrm{CPz})$.

For simplicity, each of the seven segments was denoted by $\mathrm{tn}$, where $\mathrm{n}$ is ordered integer values from $\mathrm{t} 1$ to $\mathrm{t} 7$. Specifically, $\mathrm{t} 1$ denotes the time-period of $2,050-2,100$ $\mathrm{ms}$ after presentation of the stimulus video $(0 \mathrm{~ms})$ and $\mathrm{t} 7$ denotes the time-period from 2,350 ms to $2,400 \mathrm{~ms}$ (Figure $3 \mathrm{a}$ ).

The energy values of theta oscillations in the selected POIs were assessed using a 4 (discrepancies: less, small, large, inconsistent) $\times 7$ (time segment: t1-t7) ANOVA in SPSS 20.0 (IBM, Somers, USA). Partial eta-squared $\left(\eta_{\mathrm{p}}^{2}\right)$ was used to represent the effect size for ANOVA and a Bayes test was used as an auxiliary means to test the effect (Figure 6). VAS questionnaire data were missing from one participant and therefore the analyses included 31 participants (See the Supplementary file).

\subsection{Results}

\subsubsection{Behavioral results}

ANOVA results showed a main effect of discrepancy on reaction time, $F_{(3,93)}=7.763, P<.001, \eta_{\mathrm{p}}^{2}=.200, \mathrm{BF}_{10}>100$ (Figure 4). Pairwise comparisons revealed significant differences between little-to-no discrepancy and large discrepancy $(P=.008$, $95 \% \mathrm{CI}[34.952,-3.845])$, between little-to-no and inconsistent discrepancy $(P=.03$, 95\% CI[-74.161,-2.588], $\left.\mathrm{BF}_{10}>3\right)$, between small and large discrepancy $(P=.001$, 95\% $\left.\mathrm{CI}[-32.463,-6.363], \mathrm{BF}_{10}>100\right)$, and between small discrepancy and inconsistent 
$\left(P=.04,95 \% \mathrm{CI}[-75.551,-1.227], \mathrm{BF}_{10}>100\right)$. There were no significant differences in reaction time between little-to no discrepancy and small discrepancy $(P=1.00,95 \% \mathrm{CI}[-$ 9.248,9.276]), nor between large discrepancy and inconsistent $(P=.599,95 \% \mathrm{CI}[-$ $12.553,50.504])$.

\subsubsection{Theta oscillation}

There were main effects of both discrepancy $\left(F_{(3,93)}=10.213, P=.003, \eta_{\mathrm{p}}^{2}=.248\right.$, $\left.\mathrm{BF}_{10}>100\right) \quad$ and $\quad$ time $\quad$ segment $\quad\left(F_{(6,186)}=147.125, \quad P<.001\right.$, $\left.\eta_{\mathrm{p}}^{2}=.826, \mathrm{BF}_{10}>100\right)$ on theta oscillation. There was also a significant interaction between discrepancy and time segment $\left(F_{(18,558)}=15.970, P<.001, \eta_{\mathrm{p}}^{2}=.340, \mathrm{BF}_{10}>100\right)$. Pairwise comparisons showed that theta oscillation during the inconsistent condition was significantly different from all other conditions at time segments $t 4, \mathrm{t} 5$, $t 6$, and $\mathrm{t} 7$ $(P \mathbf{S}<.05)$. A paired sample t-test showed that theta oscillations were significantly different in the little-to-no discrepancy condition compared to the large discrepancy condition at time segments $\mathrm{t} 5, \mathrm{t} 6$, and $\mathrm{t} 7(P \mathbf{s}<.05)$; and theta oscillations were also significantly different in the small compared to large discrepancy conditions at t6 and 77 $\left(P_{\mathbf{S}}<.05\right.$, Figure 5).

\subsection{Discussion of Experiment 2}

The results of Experiment 2 showed that when discrepancy was low (i.e., less than $50 \%$ ), coping strategies were similar to the consistent situation, with similar response times and theta activity. Furthermore, as the discrepancy increased, the response time significantly increase, along with an increase in theta activity, suggesting that coping 
strategy was modulated by the degree of discrepancy.

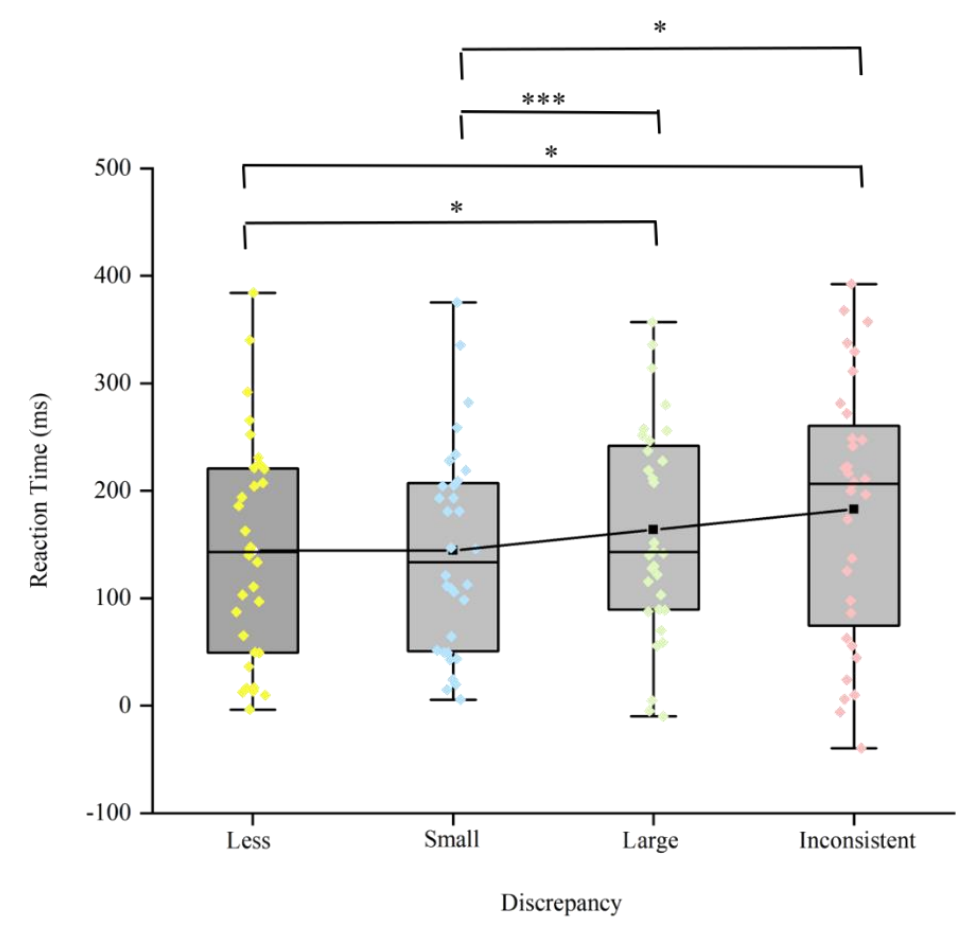

Figure 4 The reaction time of participants at different discrepancy conditions. Error bars represent standard error of the mean. $* P<0.05, * * * P<0.001$.

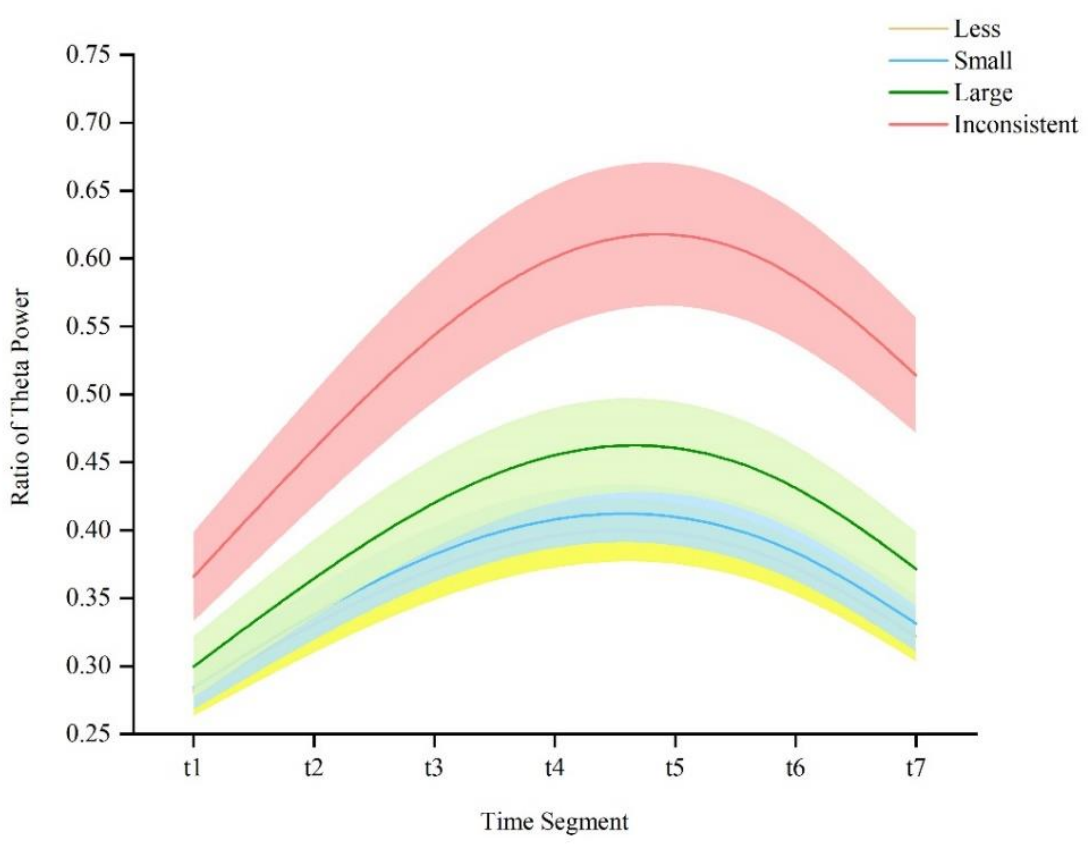

Figure 5 Changes in the EEG power spectrum at seven time-segments. The dynamic changes in 
magnitude of the theta oscillations are represented as a function of time.

(a)
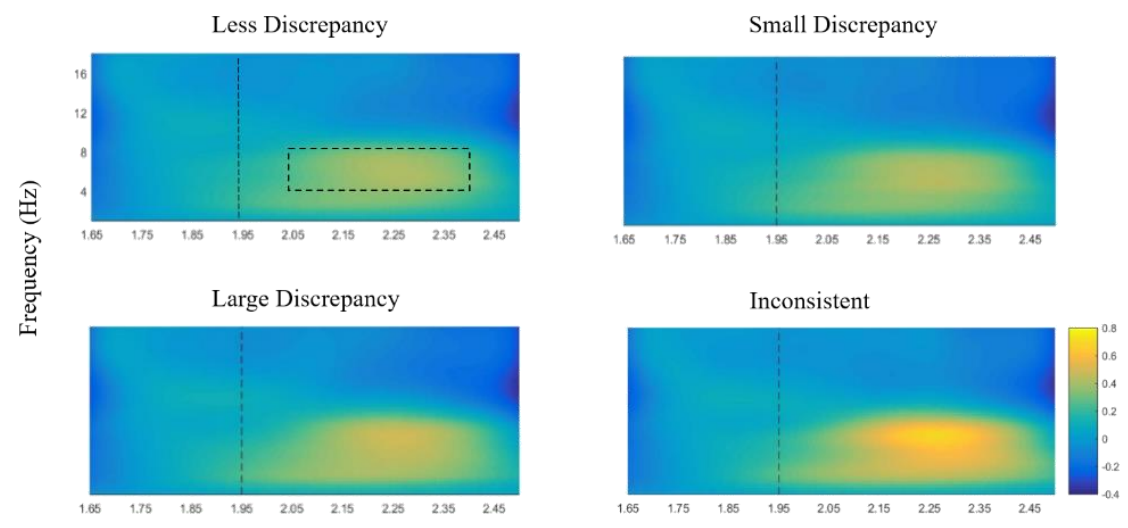

Time (s)

(b)

Less Discrepanc
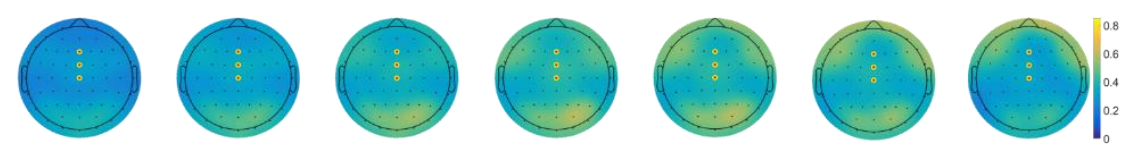

Small Discrepancy
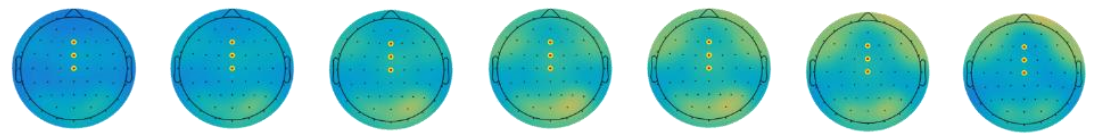

Large Discrepancy
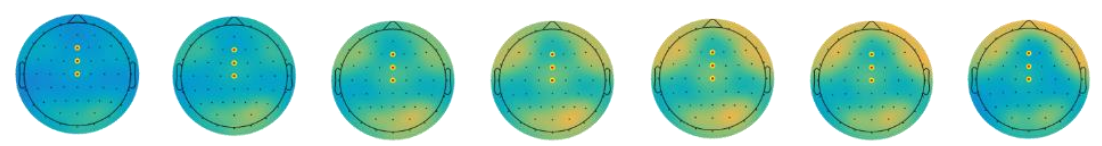

Inconsistent

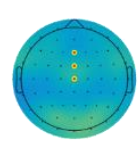

t1

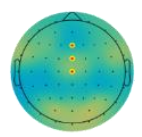

t2

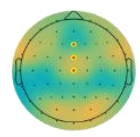

t3

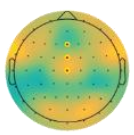

t4

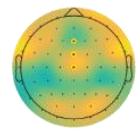

t5

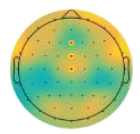

t6

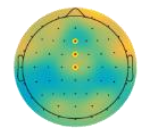

t7

Time Segment

Figure 6 (a) Time-frequency representation. They represent the average of the scalograms from Fz,

$\mathrm{FCz}$ and $\mathrm{Cz}$, where a rectangle with dashed border designates the POI from which the theta power spectrum was computed. These plots reveal that, in the inconsistent condition, the experts have greater theta power than other conditions. (b) Topographic. From left to right, the corresponding mean power spectrums in each movement segment (from $\mathrm{t} 1$ to t7) were plotted. These topographic maps reveal that the experts have stronger midfrontal theta in the inconsistent condition than others.

\section{General discussion}


The current study explored the cognitive processing characteristics of table tennis players related to discrepancy between predicted and actual outcomes. Our study design included seven discrepancy conditions, ranging from consistent ( $0 \%$ discrepancy) to inconsistent (100\% discrepancy). In Experiment 1 we found experts to be significantly more effective at processing discrepancies compared to novices. In Experiment 2, we explored the specific behavior and underlying brain activity of experts during the discriminatory task.

The superior performance on the discrimination task by experts indicates that motor experience promotes discrepancy perception. These results are consistent with previous studies demonstrating that long-term training helps to form unique cognitive processing that benefits the prediction of actions (Dicks, Davids, \& Button, 2010; Farrow, Chivers, Hardingham, \& Sachse, 1998). It is well known that motor areas activated in response to observing an action are the same as those activated when an action is actually made. Furthermore, the mere knowledge of an upcoming action is enough to activate the motor system, allowing the prediction of the outcome of another's action (Calvo-Merino, et.al., 2005). It has been postulated that experience with observed movements and action representations facilitates prediction and recognition of actions (Markus, Graf, \& Bianca, et al., 2007). Due to arduous training, table tennis experts are able to establish effective internal forward models according to existing knowledge and experience, thus improving the ability to predict the landing point of the ball and promote identification of discrepancies between predicted and actual outcomes. Novices without experience are less able to identify discrepancy than experts because they are 
unable to understand actions through internal simulations.

Importantly, the current study indicates that experts are skilled not only in the identification of discrepancies in predicted and actual outcomes but also in the identification of subtle differences in degree of discrepancy. This finding is consistent with the PRO model (Alexander \& Brown, 2011). Neural activity in the medial prefrontal cortex is constantly changing with the increasing of the degree of discrepancy. In the current study, the highest discrepancy condition (100\% inconsistent) induced high theta oscillation. Interestingly, large discrepancy conditions (i.e., 66\% and 83\%) affected response time, but did not significantly alter neural activity. The smallest discrepancy conditions $(<50 \%)$ did not significantly affect behavioral performance or brain activity. These strategies benefit experts in coping with situations where predicted outcomes do not match actual outcomes. After long-term training, athletes develop effective cognitive skills and adopt different coping strategies for outcome discrepancies. Novices on the other hand, could not distinguish more subtle differences in discrepancies and could only classify outcomes as consistent or inconsistent due to lack of specialized skill training and prior experience.

Previously, we found heightened theta oscillations in response to incongruent stimuli, indicating that theta waves are sensitive to mismatched stimuli (Lu.et.al., 2019). This result further supports the PRO model, which postulates that frontal cortex theta waves are reactive to the processing of inconsistent stimuli (Alexander \& Brown, 2011). The central frontal cortex is an important region for high-level cognitive processing, and theta oscillations within this area have been implicated in the monitoring and 
resolution of conflicting information and the occurrence of unexpected events (Moore, Gale, Morris, \& Forrester, 2006; Moore, Mills, Marshman, \& Corr, 2012). Activation of theta waves in the frontal cortex indicates the need for heightened cognitive control (Cohen \& Michael, 2014). Some research has suggested that the significant changes in neural activity caused by incongruence between predicted and actual outcomes are due to the unexpected events that cause behavioral switching in participants (Hikosaka, \& Isoda, 2010). In Experiment 2, participants predicted the landing point of a ball according to observed body movements made by the server and reacted with corresponding behavioral preparations. However, through experimental manipulation, the flight trajectory after racquet-ball contact was inconsistent with predicted behavior, and as such, it was necessary for the participant to alter their action plan to be consistent with the actual outcome. Behavioral switching requires high cognitive cost (Masaki, Isoda, Okihide, \& Hikosaka, 2007). The current study found that athletes faced with a large discrepancy between predicted and actual outcomes demonstrated an increased response time with no significant alterations in neural activity, suggesting a lower cognitive cost. Table tennis is a time-constrained sport with limited information, requiring athletes to make judgments in both time and space. A reduction in cognitive cost is beneficial for table tennis athletes and is present after long-term specialized training. Hung et al. (2014) explained that experts respond quickly to unexpected events because they have cognitive resource reserves to cope with discrepancy between predicted and actual outcomes. Athletes therefore, use less cognitive resources to cope with discrepancy than novices, leading to a more efficient processing pattern. Consistent with the 
current study results and the PRO model, it is only necessary for athletes to activate additional cognitive resources when the discrepancy between predicted and actual outcomes is inconsistent $(100 \%)$. These data indicate that the allocation of cognitive resources is one reason for superior performance in athletes.

The presented study provides further evidence for the applicability of the PRO model in the understanding of another's actions. The PRO model has been validated in classical tasks such as the change signal task (Brown, 2009) and the Eriksen Flanker task(Scheffers \& Coles, 2000). Behavioral and neural research has shown that these two tasks can induce regulation of cognitive resources and changes in frontal cortex activity (Alexander \& Brown, 2011). Notably, both tasks involve only the participants themselves, without judging the actions of another. The participants in this experiment were in the perspective of observers, and needed to observe and anticipate the actions of others, which was different from the tasks mentioned above. Current result also consistent with the PRO model and supports the feasibility of the model in understanding others' action.

We found significant differences in theta oscillations at $\mathrm{t} 4$, indicating that participants had processed enough visual information to update their action prediction. Compared with the smaller discrepancies, neural activity was greater under the inconsistent condition, indicating that when the discrepancy between predicted and actual outcomes increased, the neural activity increased.

Taken together, the present study confirmed that athletes develop effective cognitive processing skills after long-term training. The results expand the two-category 
consistent-inconsistent model to four subtle and gradual categories of outcomes, laying a foundation for further research on discrepancy detection. Specifically, greater discrepant stimuli require more cognitive resources for behavioral control. Consistent with previous studies, additional cognitive resources were associated with increased theta oscillations. Lastly, the current study validated the PRO model and expanded its application to the field of understanding others' actions.

\section{References}

Alexander, W.H., \& Brown, J. W. (2011). Computational models of performance monitoring and cognitive control. Topics in cognitive science,2(4), 658-677. https://doi.org/10.1111/j.1756-8765.2010.01085.x

Alexander, W. H., \& Brown, J. W. (2012). Medial prefrontal cortex as an action-outcome predictor. Nature Neuroscience, 14(10), $1338-1344$. https://doi.org/10.1038/nn.2921

B. Calvo-Merino, D.E. Glaser, J. Grèzes, R.E. Passingham, P. Haggard. (2005). Action Observation and Acquired Motor Skills: An fMRI Study with Expert Dancers. Cerebral Cortex, 15(8), 1243-1249. https://doi.org/10.1093/cercor/bhi007

Benguigui, N., \& Ripoll, H. (1998). Effects of tennis practice on the coincidence timing accuracy of adults and children. Research Quarterly for Exercise and Sport, 69, 217-223. https://doi.org/10.1080/02701367.1998.10607688

Brown, J. W. (2009). Conflict effects without conflict in anterior cingulate cortex: multiple response effects and context specific representations. Neuroimage, 47(1), 
334-341. https://doi.org/10.1016/j.neuroimage.2009.04.034

Cavanagh, J. F., Cohen, M. X., \& Allen, J. (2009). Prelude to and resolution of an error: eeg phase synchrony reveals cognitive control dynamics during action monitoring. Journal of Neuroscience,29(1), 98-105. https://doi.org/10.1016/j.neuroimage.2009.04.034

Cavanagh, J. F., \& Frank, M. J. (2014). Frontal theta as a mechanism for cognitive control. Trends in Cognitive Sciences, 18(8), 414-421. https://doi.org/10.1523/JNEUROSCI.4137-08.2009

Cohen, \& Michael, X. (2014). A neural microcircuit for cognitive inconsistent detection and signaling. Trends in Neurosciences, 37(9), 480-490.

Denis, D., Rowe,R., Williams, A.M., \& Milne, E.(2017).The role of cortical sensorimotor oscillations in action anticipation. Neuroimage, 146, 1102-1114. https://doi.org/10.1016/j.neuroimage.2016.10.022

Dicks, M., Davids, K., \& Button, C. (2010). Individual differences in the visual control of intercepting a penalty kick in association football. Human Movement Science, 29(3), 401-411. https://doi.org/10.1016/j.humov.2010.02.008

Farrow, D., Chivers, P., Hardingham., C., \& Sachse, S. (1998). The effect of videobased perceptual training on the tennis return of serve. International Journal of Sport Psychology, 29, 231-242.

Guy, C., P Géraldine, Julian, C., Axelle, L., Anita, C., \& Carlos, C., et al. (2016). Brain oscillations in sport: toward eeg biomarkers of performance. Frontiers in Psychology, 7. https://doi.org/10.3389/fpsyg.2016.00246 
Helm, F., Caal-Bruland, R., Mann, D. L., Troje, N. F., \& Munzert, J. (2019). Integrating situational probability and kinematic information when anticipating disguised movements. Psychology of Sport and Exercise, 46, 101607. https://doi.org/10.1016/j.psychsport.2019.101607

Hikosaka, O., \& Isoda, M. (2010). Switching from automatic to controlled behavior: cortico-basal ganglia mechanisms. Trends in Cognitive Sciences, 14(4), 154-161. https://doi.org/10.1016/j.tics.2010.01.006

Hung, T. M., Spalding, T. W., Santa Maria, D. L., \& Hatfield, B. D. (2004). Assessment of reactive motor performance with event-related brain potentials: Attention processes in elite table tennis players. Journal of Sport and Exercise Psychology, 26(2), 317-337. https://doi.org/10.1123/jsep.26.2.317

Körding, K. (2007). Decision theory: what" should" the nervous system do?. Science, 318(5850), 606-610. https://doi.org/10.1126/science.1142998

Lu,Y., Yang,T., Hatfield, B. D., Cong, F., \& Zhou,C. (2019).Influence of cognitive-motor expertise on brain dynamics of anticipatory-based outcome processing. Psychophysiology, 57(10). https://doi.org/10.1111/psyp.13477

Macmillan, N. A., \& Kaplan, H. L. (1985). Detection theory analysis of group data: estimating sensitivity from average hit and false-alarm rates. Psychological Bulletin, 98(1), 185-99. https://doi.org/10.1037/0033-2909.98.1.185

Markus, Graf, and, Bianca, Reitzner, \& and, et al. (2007). Predicting point-light actions in real-time. Neuroimage. https://doi.org/10.1016/j.neuroimage.2007.03.017

Masaki, Isoda, Okihide, \& Hikosaka. (2007). Switching from automatic to controlled 
action by monkey medial frontal cortex. Nature Neuroscience. https://doi.org/10.1038/nn1830

Michael.J.Wright, Daniel.T.Bishop, Robin.C.Jackson \& Bruce Abernethy. (2013). Brain regions concerned with the identification of deceptive soccer moves by higher-skilled and lower-skilled players. Frontiers in Human Neuroscience, 7,1-15. https://doi.org/10.3389/fnhum.2013.00851

Moore, R. A., Mills, M. , Marshman, P., \& Corr, P. J. (2012). Behavioural inhibition system (bis) sensitivity differentiates eeg theta responses during goal conflict in a continuous monitoring task. International journal of psychophysiology: official journal of the International Organization of Psychophysiology, 85(2), 135-144. https://doi.org/10.1016/j.ijpsycho.2012.06.006

Moore, R. A., Gale, A., Morris, P. H., \& D Forrester. (2006). Theta phase locking across the neocortex reflects cortico-hippocampal recursive communication during goal conflict resolution. International Journal of Psychophysiology, 60(3), 260-273. https://doi.org/10.1016/j.ijpsycho.2005.06.003

Murray, N.P., \& Hunfalvay, M. (2016). A comparison of visual search strategies of elite and non-elite tennis players through cluster analysis. Journal of Sports Sciences, 1-6. https://doi.org/10.1080/02640414.2016.1161215

Romain, G., \& Arnaud, D. (2011). Single-trial normalization for event-related spectral decomposition reduces sensitivity to noisy trials. Frontiers in Psychology, 2. https://doi.org/10.3389/fpsyg.2011.00236

Rowe, R., Poulter, D.M., and McKenna, F.P. (2009). The effect of disguise on novice 
and expert tennis players' anticipation ability. J.Appl.Sport Psychol. 21,178-185. https://doi.org/10.1080/10413200902785811

Runigo, C.L., Benguigui, N., \& Bardy, B.G. (2005). Perception-action coupling and expertise in interceptive actions. Human Movement Science, 24(3), 429-445. https://doi.org/10.1016/j.humov.2005.06.008

Scheffers, M. K., \& Coles, M. G. (2000). Performance monitoring in a confusing world: Error-related brain activity, judgments of response accuracy, and types of errors. Journal of Experimental Psychology: Human Perception and Performance, 26(1), 141-151. https://doi.org/10.1037//0096-1523.26.1.141

Vicario, C. M., Makris, S., \& Urgesi, C. (2016). Do experts see it in slow motion? altered timing of action simulation uncovers domain-specific perceptual processing in expert athletes. Psychological Research, 81(6), 1-12. https://doi.org/10.1007/s00426-016-0804-z

Ward, P., Williams, A. M., \& Bennett, S. J. (2002). Visual search and biological motion perception in tennis. Res $Q$ Exerc Sport, 73(1), 107-112. https://doi.org/10.1080/02701367.2002.10608997

Warren-Westgate, L. S., Jackson, R. C., Blenkinsop, G. M., \& Hiley, M. J. (2021). Earlier detection facilitates skilled responses to deceptive actions. Human Movement Science, 80, 102885. https://doi.org/10.1016/j.humov.2021.102885

Wimshurst, Z.L., Sowden,P.T., \& Wright, M. (2016). Expert-novice differences in brain function of field hockey players. Neuroscience. https://doi.org/10.1016/j.neuroscience.2015.11.064 
Zhao,Q., Lu,Y., Jaquess, K. J., \& Zhou, C. (2018). Utilization of cues in action anticipation in table tennis players. Journal of Sports Sciences, 36(23), 1-7. https://doi.org/10.1080/02640414.2018.1462545 